\title{
On a certain Class of Linear Substitutions with Common Invariants, and an Associated Substitution of Order Four.
}

\author{
By D. G. TAYLoR.
}

(Received 21st April 1918. Read 9th May 1913).

\section{Section I.-A Class of Linear Substitutions with Common Invariants.}

\$1. The determinant

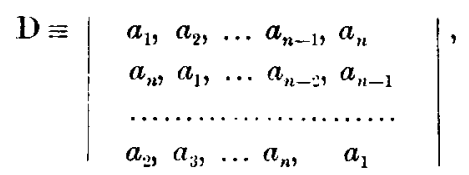

each row of which contains the same $n$ elements in the same cyclic order, with ' $a_{1}$ always in the leading diagonal, is the product of $n$ linear factors, ${ }^{*}$ which we shall write as follows:

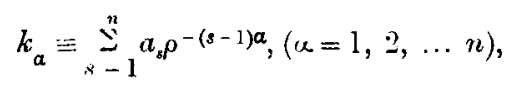

where $\rho$ is any primitive $n^{\text {th }}$ root of unity. That $k_{a}$ is a factor of $\mathrm{D}$ is at once evident on multiplying the respective columns after the first by $\rho^{-\alpha}, \rho^{-2 \alpha}, \ldots \rho^{-(n-1) \alpha}$, and the respective rows after the first by the same quantities in reversed order, and then adding all the rows (or columns) together.

Consider the linear substitution of which $\mathrm{D}$ is the determinant :

$$
\begin{aligned}
& x_{1}^{\prime}=a_{1} x_{1}+a_{2} x_{2}+\ldots+a_{n} x_{n} \\
& x_{2}^{\prime}=a_{n} x_{1}+a_{1} x_{2}+\ldots+a_{n-1} x_{n} \\
& x_{n}^{\prime}=a_{2} x_{1}+a_{3} x_{2}+\ldots+a_{1} x_{n}
\end{aligned}
$$

* See Scott, Determinants, p. 81. 
Form its characteristic equation

\section{by putting}

$$
f(k)=0
$$

$$
\boldsymbol{x}_{\beta}^{\prime}=k x_{\beta},(\beta=1,2, \ldots n),
$$

and eliminating the $x_{\beta}$. The roots of $f(k)$ are clearly the above $k_{\alpha}$; and on substituting $k_{\alpha} x_{\beta}$ for $x_{\beta}^{\prime}$ in equations (1) we find that they are satisfied, provided

$$
\frac{x_{1}}{\rho^{-\alpha}}=\frac{x_{2}}{\rho^{-2 a}}=\ldots=\frac{x_{n-1}}{\rho^{-(n-1) a}}=\frac{x_{n}}{1} .
$$

These values of $x_{1}, \ldots x_{n}$ define an invariant point* or pole $\mathrm{P}_{\alpha}$ of the substitution. By varying a we obtain $n$ poles $\mathbf{P}_{1}, \mathbf{P}_{2}, \ldots \mathbf{P}_{n}$, forming a complete set of poles, with their coordinates entirely independent of the coefficients $a_{r}$. With the one restriction that $\mathrm{D}$ must not vanish, the $a_{r}$ may have any real or complex values, and every substitution of the form (1) possesses the common system of invariant points $P_{1}, \ldots P_{n}$.

The corresponding linear invariants, or invariant $(n-2)$ planes, also possessed in common, are easily seen to be

$$
\xi_{\alpha} \equiv \stackrel{\beta}{n=1}_{\beta=1}^{n} \rho^{\alpha \beta} x_{\beta}=0,(\alpha=1, \underline{\varrho}, \ldots n)
$$

$\$ 2$. The result of successive applications of substitutions of the class (1), whether alike or different, is always another substitution of the class, the multipliers (or roots of the characteristic equation) for the resultant being the products of corresponding multipliers for the components. The substitution inverse to (1) is also of the same class.

There will be differences of type + within the class, according to the system of equalities among the multipliers. The minimum system of invariant points and $(n-2)$-planes, viz. the $\mathrm{P}_{a}$ and the $\xi_{a}$, belongs to all : but these will not exhaust the invariant points and $(n-2)$-planes of a substitution having one or more equalities among

* Linear Substitutions and their Invariants, §2. Proc. Edin. Muth. Soc., Vol. XXX.

+ lbid. $\$ 9$. 
its multipliers. Such a substitution will have at least one line of poles, and at least one pencil of invariant $(n-2)$-planes.

Given the $\mathrm{P}_{a}$ (or the $\xi_{a}$ ) and the corresponding multipliers, we can build up the substitution uniquely. If the mutual ratios of the multipliers are $r^{\text {th }}$ roots of unity; the substitution will be of order $r$. (Ibid. §5.) We can thus construct substitutions of the class (1) of any order we please.

Section II.-The Associated Substitution of Order Four $S(\rho)$.

$\$ 3$. Consider the substitution which transforms the frame of reference into the invariant frame of (1). Writing $x^{\prime}$ in place $\xi$ in $(2)$, and introducing the numerical factor $n^{-\frac{1}{2}}$, taken always with the positive sign, we express it in the form

$$
x^{\prime}=n^{-\frac{1}{2}} \sum_{\beta=1}^{n} \rho^{\alpha \beta} x_{\beta},(\alpha=1,2, \ldots n)
$$

The introduction of the factor $n^{-\frac{1}{2}}$ reduces the modulus of the determinant of (3) to unity, as will appear shortly. We shall denote this substitution by $\mathbf{S}(\rho)$, the left-hand member of its characteristic equation by $\Delta_{n}(o, k)$, and its determinant by $\Delta_{n}(\rho, 0)$ or simply $\Delta_{n}(\rho)$.

Thus, with $k=n^{-\frac{1}{k}}$,

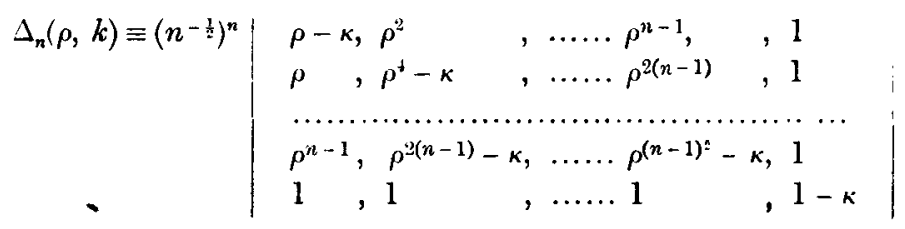

To obtain the substitution inverse to $S(\rho)$ multiply both sides of (3) by $\rho^{\gamma \alpha}$, and add for the $n$ values of a. The coefficient of $x_{\beta}$ on the right is

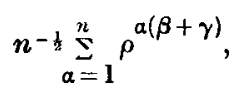

which differs from zero only in the case of $\beta=n-\gamma$, and then takes the value $u^{\frac{1}{3}}$. Hence

$S^{-1}(\rho) . \quad \quad x_{n-\gamma}=n^{-1} \stackrel{\Sigma}{n}_{a=1}^{n} \rho^{\gamma a} x_{a}^{\prime},(\gamma=1,2, \ldots n)$,

where $x_{0}$ is regarded as equivalent to $x_{n}$. 
The only difference between (4) and (3) is that on the left of (4) the order of the first $(n-1)$ variables $x_{1}, \ldots x_{n-1}$ is reversed.

Now let $x^{\prime \prime}{ }_{\gamma}(\gamma=1,2, \ldots n)$ be the result of two applications of $S(\rho)$. Then

$\mathbf{S}^{2}(\rho)$.

$$
\begin{array}{rlr}
x_{\gamma}^{\prime \prime} & =n-\frac{1}{\beta} \sum_{\beta=1}^{n} \rho^{\gamma \beta} x_{\beta}^{\prime} \text { by }(3), \\
& =x_{n-\gamma} \quad \text { by }(4) ;
\end{array}
$$

the original variables being restored, but, with the exception of the last, which remains unchanged, reversed in order. It follows that $\mathbf{S}^{4}(\rho)$ is equivalent to the identical substitution, and that $\mathrm{S}(\rho)$ is of order four. Further, since the multiplier with which the variables are restored is unity, we have

$$
\begin{gathered}
\Delta_{n}^{4}(\rho)=1, \\
\Delta_{n}(\rho)=\exp \left(\frac{1}{2} p \pi i\right),
\end{gathered}
$$

$p$ having one of the values $0,1,2,3$.

$\$ 4$. $S(\rho)$ being of order four, the roots of its characteristic equation must be fourth roots of unity $(I b i d, \$ 5)$, whence

$$
\Delta_{n}(\rho, k) \equiv(-)^{n}(k-1)^{q}(k+1)^{r}(k-i)^{\prime}(k+i)^{t},
$$

where

$$
q+r+s+t=n
$$

In finding the indices, we shall confine ourselves to a specific value of $\rho$,

$$
\rho=\exp (2 \pi i / n) \text {. }
$$

The more general case need not detain us, for other values of $\rho$ lead to the same quantities $x_{a}^{\prime}$ in (3), only in a different order.

By multiplication of determinants we find for

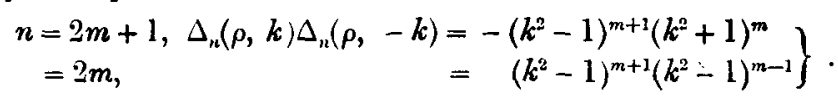

By a theorem of Gauss (see Mathews, Theory of Numbers, §184), writing

$$
\mathrm{S}=\sum_{s=0}^{a-1} \exp \left(2 s^{2} \pi i / n\right)
$$

we have for

$$
\begin{array}{rlrr}
n & \equiv 0(\bmod 4), & \mathrm{S} & =(1+i) \\
& \equiv 1 & & \sqrt{n} \\
& \equiv 2 & & \sqrt{n} \\
& \equiv 3 & & 0 \\
& & & i \sqrt{ } n .
\end{array}
$$


Now the coefficient of $k^{n-1}$ in $\Delta_{n}(\rho, k)$ is

$$
(-)^{n-1} n^{-\frac{1}{2} S} \text {; }
$$

and with $n=2 m+1, \Delta_{n}(\rho, k)$ must by ( 7$)$ have the form

$$
\Delta_{n}(\rho, k)=-(k-1)^{\dot{\alpha}}(k+1)^{m+1-\alpha}(k-i)^{\beta}(k+i)^{m-\beta} \text {. }
$$

Picking out the coefficient of the second-highest power of $k$, we have for

$$
\begin{array}{ll}
n \equiv 1(\bmod 4), & (2 \alpha-m-1)+i(2 \beta-m)=1, a=\frac{1}{2} m+1, \beta=\frac{1}{2} m ; \\
n \equiv 3 \quad(2 \alpha-m-1)+i(2 \beta-m)=i, a=\frac{1}{2}(m+1)=\beta .
\end{array}
$$

Again with $n=2 m, \Delta_{n}(\rho, k)$ is of form

whence for

$$
\Delta_{n}(\rho, k)=(k-1)^{a}(k+1)^{m+1-\alpha}(k-i)^{\beta}(k+i)^{m-1-\beta},
$$

$$
\begin{array}{rlrl}
n & \equiv 0(\bmod 4), & a=\frac{1}{2} m+1, \beta=\frac{1}{2} m ; \\
& \equiv 2 & a=\frac{1}{2}(m+1), \beta=\frac{1}{2}(m-1) .
\end{array}
$$

The following table summarises these results, and also gives the values of $p$ in (5):

\begin{tabular}{|c|c|c|}
\hline$n$ & $\Delta_{n}(\rho, k)$ & $p \equiv(\bmod 4)$ \\
\hline $4 s$ & $(k-1)^{s+1}(k+1)^{s}(k-i)^{s}(k+i)^{s-1}$ & $2 s+1$ \\
\hline $4 s+1$ & $-(k-1)^{s+1}(k+1)^{a}(k-i)^{\prime}(k+i)^{s}$ & $2 s$ \\
\hline $4 s+2$ & $(k-1)^{s+1}(k+1)^{++1}(k-i)^{s}(k+i)^{s}$ & $2 s+2$ \\
\hline $4 s+3$ & $-(k-1)^{s+1}(k+1)^{s+1}(k-i)^{s+1}(k+i)^{a}$ & $2 s+3$ \\
\hline
\end{tabular}

$\$ 5$. Taking $q, r, s, t$ as in (6), the canonical form of $\mathrm{S}(\rho)$ is clearly the following:

$$
\begin{array}{ll}
\xi_{a}^{\prime}=\xi_{a}, & (a=1,2, \ldots \ldots q), \\
\xi_{\beta}^{\prime}=-\xi_{\beta}, & (\beta=q+1, \ldots \ldots q+r), \\
\xi_{\gamma}^{\prime}=i \xi_{\gamma}, & (\gamma=q+r+1, \ldots \ldots q+r+s), \\
\xi_{\delta}^{\prime}=-i \xi_{\delta}, & (\delta=q+r+s+1, \ldots \ldots n),
\end{array}
$$

the $\xi$ being a set, not in general unique, of linear invariants of $S(\rho)$. It remains to express the $\xi$ in terms of the original coordinates $x$. 
A separation can be made between the $\xi$ with real and those with imaginary multipliers; the former appearing as the invariants of a new substitution in $(q+r)$ variables, and the latter in the same relation to another substitution in $(s+t)$ variables. The reduction shows that the $\xi$ are in all cases real linear functions of the $x$; thus the linear invariants, and therefore also the poles of $\mathrm{S}(\rho)$ are all real.

An invariant of $S(\rho)$ with real multiplier $( \pm 1)$ is an invariant of $S^{2}(\rho)$ with multiplier +1 , and therefore by (4) is unaltered by interchange throughout of $x_{\alpha}, x_{n-a},(\alpha=1,2, \ldots, n-1)$. An invariant of $\mathrm{S}(\rho)$ with imaginary multiplier $( \pm i)$ is an invariant of $\mathbf{S}^{2}(\rho)$ with multiplier -1 , and therefore simply changes sign on interchange throughout of $x_{\alpha}, x_{n-a}$.

Write

$$
\begin{aligned}
& x_{a}+x_{n-a}=y_{a}, \\
& x_{\alpha}-x_{n-a}=z_{a},
\end{aligned}
$$

where $a$ takes all integral values from 1 to $m$ or $m-1$ according as $n=2 m+1$ or $2 m$. In the latter case write $x_{m}=y_{m}$, and in both cases $x_{n}=y_{m+1}$. Then the invariants with real multipliers are linear functions of the $y$, those with imaginary multipliers of the $z$.

But putting

$$
\left.\begin{array}{rl}
\rho^{\alpha}+\rho^{-\alpha} & \equiv 2 \cos (2 \pi a / n)=\sigma_{a} \\
-i\left(\rho^{\alpha}-\rho^{-\alpha}\right) & \equiv 2 \sin (2 \pi \alpha / n)=\tau_{\alpha}
\end{array}\right\}
$$

and taking

(i) $n=2 m+1$, we have from (3),

and

$$
\left.\begin{array}{rl}
\sqrt{ } n \cdot y_{a}^{\prime} & =\sum_{\beta=1}^{m} \sigma_{a \beta} y_{\beta}+2 y_{m+1},(a=1,2 \ldots m) \\
\sqrt{ } n \cdot y_{m+1}^{\prime} & =\sum_{\beta=1}^{m} y_{\beta}+y_{m+1}
\end{array}\right\}
$$

$$
\sqrt{ } n \cdot z^{\prime}=\underset{\beta=1}{\stackrel{m}{\sum^{\prime}} i \tau_{\alpha \beta} z_{\beta}},(\alpha=1,2, \ldots m)
$$

The former is a substitution of order 2 in the $(m+1)$ variables $y_{1} \ldots y_{m+1}$, of which the invariants, when expressed in terms of the $x$, are those of $(3)$ with real multipliers, and have real coeflicients throughout. The invariants of (10) are likewise identical with 
those of (3) which have imaginary multipliers; and since, if we omit the $i$ on the right-hand side of (10), the only adjustment required is to alter the multipliers from $\pm i$ to \pm 1 respectively, the invariants themselves remaining unchanged, it is clear that they are real functions of the $z$, and hence also of the $x$.

(ii) Similarly for $n=2 m$. Equations (9) are replaced by

$$
\begin{aligned}
& \sqrt{n} \cdot y_{a}^{\prime}=\sum_{\beta=1}^{m-1} \sigma_{a \beta} y_{\beta}+(-)^{a} 2 y_{m}+2 y_{m+1},(\alpha=1, \ldots m-1) \\
& \sqrt{n} \cdot y_{m}^{\prime}=\sum_{\beta=1}^{m-1}(-)^{\beta} y_{\beta}+(-)^{m} y_{m}+y_{m+1}, \\
& \sqrt{ } n \cdot y_{m+1}^{\prime}=\sum_{\beta=1}^{m-1} \cdot y_{\beta}+y_{m}+y_{m+1}
\end{aligned}
$$

and (10) by

$$
\sqrt{ } n \cdot z_{\alpha}^{\prime}=\sum_{\beta=1}^{m-1} i \tau_{\alpha \beta} z_{\beta},(\alpha=1, \ldots m-1)
$$

On comparing the forms assumed by the characteristic equation in (9) to (12) with those of the table of $\$ 4$, identities are obtained, of which the following may be noted:

With $n=2 m+1=4 s+1$, the $\sigma, \tau$ having the values defined in $(8)$,

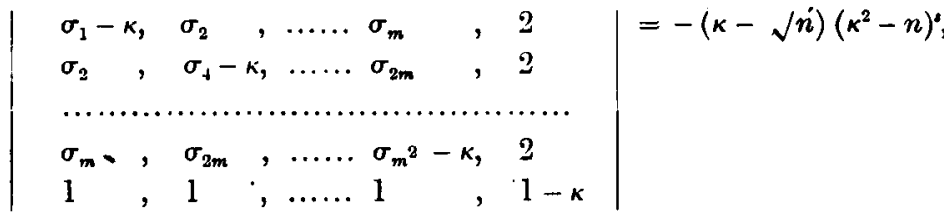

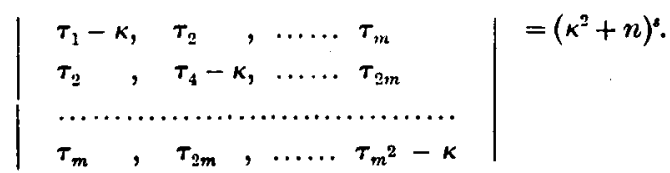

The invariants $\xi$ obtained by the help of (9)-(12) as far as $n=8$, are tabulated below. Only those with multipliers $+1,+i$ are written down; those with multipliers $-1,-i$ are deduced therefrom by changing the sign of $\sqrt{ } n$ wherever it occurs explicitly. In the cases of two or more invariants with the same 
multiplier, those given are not unique, and any linear functions of them would serve equally well. The form

$$
\sum_{a=1}^{n} x_{\alpha}+\sqrt{ } n \cdot x_{n}
$$

which appears first in each set, is at once seen to be invariant from (3). It is to be remembered that the $\sigma, \tau$ depend on the value of $n$.

Since the determinant of (3) is symmetrical, the pole corresponding to any invariant

$$
\sum_{a=1}^{n} p_{a} x_{a}
$$

can be immediately deduced: it is in fact the point with coordinates $\left(p_{1}, p_{2} \ldots p_{n}\right)$. The extension to the case of equal multipliers is easily made.

Relations come to light in the process of finding the forms, which are exemplified in the case of $n=7$. For this case the substitution (9) will have as an invariant with multiplier +1 the function

$$
\sum_{a=1}^{4} p_{a} y_{a}
$$

provided

$$
\begin{array}{rcrr}
-\left(\sqrt{ } 7-\sigma_{1}\right) p_{1}+ & \sigma_{2} p_{2}+ & \sigma_{3} p_{3}+ & p_{4}=0 \\
\sigma_{2} p_{1}-\left(\sqrt{ } 7-\sigma_{3}\right) p_{2}+ & \sigma_{1} p_{3}+ & p_{4}=0 \\
\sigma_{3} p_{1}+ & \sigma_{1} p_{2}-\left(\sqrt{ } 7-\sigma_{2}\right) p_{3}+ & p_{4}=0 \\
2 p_{1}+ & 2 p_{2}+ & 2 p_{3}-(\sqrt{ } 7-1) p_{4} & =0 .
\end{array}
$$

Since there are two invariants of this kind, only two of these four equations can be independent; in other words, each first minor of the determinant of the coefficients is zero. This can be verified in virtue of the relations

$$
\begin{gathered}
\sigma_{1}+\sigma_{2}+\sigma_{3}=-1, \\
\sigma_{2} \sigma_{3}+\sigma_{3} \sigma_{1}+\sigma_{1} \sigma_{2}=-2 ;
\end{gathered}
$$

and the statement remains true when the sign of the radical is changed.

Similarly the conditions that

$$
\stackrel{a}{a=1}_{a}^{3} p_{a} z_{a}
$$

be an invariant of (10) for this case, with multiplier $+i$, are 


$$
\begin{aligned}
& -\left(\sqrt{ } \boldsymbol{\tau}-\tau_{1}\right) p_{1}+\quad \tau_{2} p_{2}+\quad \tau_{3} p_{3}=0 \\
& \tau_{2} p_{1}-\left(\sqrt{ } 7+\tau_{3}\right) p_{2}-\quad \tau_{1} p_{3}=0 \\
& \tau_{3} p_{1-} \quad \tau_{1} p_{2}-\left(\sqrt{ } 7-\tau_{2}\right) p_{3}=0,
\end{aligned}
$$

only one of which can be independent. This is evident on remembering that

$$
\begin{gathered}
\tau_{1}+\tau_{2}-\tau_{3}=\sqrt{ } 7, \\
\tau_{2} \tau_{3}+\tau_{3} \tau_{1}-\tau_{1} \tau_{2}=0,
\end{gathered}
$$

but is no longer true when the sign of the radical in the three conditions is changed.

$\$ 6$. The application of the substitution (3) to the transformation of algebraic equations may be indicated. The equations

$$
\begin{aligned}
& \phi_{n}(x) \equiv \prod_{a=1}^{n}\left(x-x_{\alpha}\right)=0, \\
& \psi_{n}(x) \equiv \prod_{a=1}^{n}\left(x-x_{a}^{\prime}\right)=0
\end{aligned}
$$

are reciprocally related, the roots of each being derived from those of the other by the same linear substitution.

- Consider the effect of increasing the roots of $\phi_{n}(x)$ by the constant quantity $\lambda$. It is clear that all the roots of $\psi_{n}(x)$ will remain unchanged, except $x_{n}^{\prime}$, which will be increased by $\sqrt{ } n . \lambda$. If therefore we choose $\lambda=-n^{-1} \sum_{a=1}^{n} x_{a}$, two things will happen :

(i) the term in $x^{n-1}$ will drop out from $\phi_{n}(x)$;

(ii) the new $x_{n}^{\prime}$ will vanish, and $\psi_{n}(x)$ be replaced by the function of degree one lower

$$
\psi_{n-1}(x) \equiv \prod_{a=1}^{n=1}\left(x-x_{a}^{\prime}\right)
$$

Were it possible to obtain the $x^{\prime}{ }_{a}$ in terms of the coefficients of $\phi_{n}(x), \phi_{n}(x)$ could be solved. In the case of the cubic

$$
\phi_{3}(x) \equiv x^{3}+3 p x+q=0,
$$

(3) reduces, after the change indicated, to the equations

$$
\begin{aligned}
x_{1}^{\prime} & =3^{-\mathrm{t}}\left(\omega x_{1}+\omega^{2} x_{2}+x_{3}\right), \\
x_{2}{ }^{\prime} & =3^{-\mathrm{t}}\left(\omega^{2} x_{1}+\omega x_{2}+x_{3}\right), \\
0=x_{3}{ }^{\prime} & =3^{-1}\left(x_{1}+x_{2}+x_{3}\right) ;
\end{aligned}
$$


63

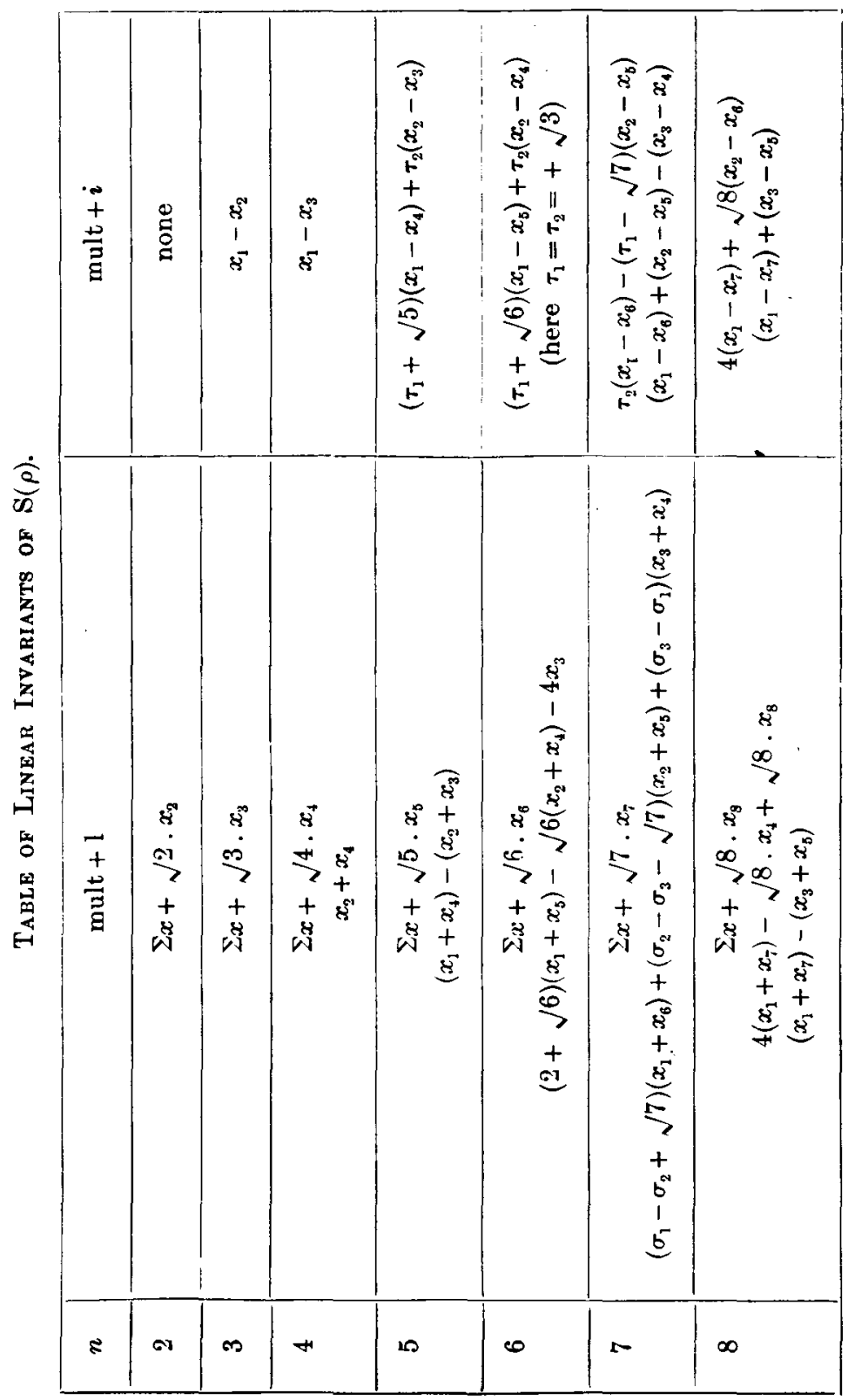


whence

$$
\begin{gathered}
x_{1}^{\prime} x_{2}^{\prime}=-\Sigma x_{2} x_{3}=-3 p, \\
x_{1}^{\prime 3}+x_{2}^{\prime 3}=3 \sqrt{ } 3 \cdot x_{1} x_{2} x_{3}=-3 \sqrt{ } 3 . q,
\end{gathered}
$$

leading to the usual solution. In the case of the biquadratic also the method leads to Euler's reducing cubic.

\section{Section III.-Triangles in Multiple Perspective. Geometrical Interpretation of Substitution (1) for $n=3$.}

$\$ 7$. The triangle $X_{1} X_{2} X_{3}$ is said to be in perspective with $Y_{\alpha} Y_{\beta} Y_{\gamma}$, the order of the vertices being significant, when $X_{1} \bar{Y}_{a}, X_{2} Y_{\beta}, X_{3} Y_{\gamma}$ meet in a point. Thus $X_{1} X_{2} X_{:}$may be in perspective with another triangle in either of six different ways, according to the order of the vertices. Let the vertices, referred to $\mathrm{X}_{1} \mathrm{X}_{2} \mathrm{X}_{3}$ as triangle of reference in any system of homogeneous point-coordinates, be

$$
\mathrm{Y}_{r}\left(x_{r 1}, x_{r 2}, x_{r 3}\right),(r=1,2,3) ;
$$

then the six cases, with their conditions, fall into two sets of three as follows: $X_{1} X_{2} X_{3}$ is in perspective with
(i) $Y_{i} Y_{2} Y_{3}$
(ii) $Y_{2} Y_{3} Y_{1}$
provided
$\left.\begin{array}{l}x_{12} x_{23} x_{31}=x_{13} x_{21} x_{32} \\ x_{11} x_{22} x_{33}=x_{12} x_{23} x_{31} \\ x_{15} x_{21} x_{32}=x_{11} x_{22} x_{33}\end{array}\right\}$
(iii) $Y_{3} Y_{1} Y_{2}$
$x_{22} x_{31} x_{13}=x_{33} x_{12} x_{21}$
(v) $Y_{3} Y_{2} Y_{1}$
$x_{33} x_{12} x_{31}=x_{11} x_{23} x_{32}$
$x_{11} x_{23} x_{32}=x_{22} x_{31} x_{13}$
(vi) $Y_{2} Y_{1} Y_{3}$
",

The -poles of perspective will be called $Z_{r}(r=1,2, \ldots 6)$. Sets (A), (B) are distinguished by the cyclic order of the vertices $Y_{1} Y_{2} Y_{3}$. In each set any two of the conditions involve the third. It is thus clear that

(a) triple perspective will occur when two conditions of one set are fulfilled, and therefore two of the vertices (say $\mathrm{Y}_{2} \mathrm{Y}_{3}$ ) may be taken at random. The three poles of perspective are clearly the vertices of a third triangle in triple perspective with each of the other two, the poles for each pair being the vertices of the remaining one;

( $\beta$ ) quadruple perspective occurs when three conditions, not all of one set, are fulfilled. The construction of such a case 
is a simple and instructive exercise in homogeneous coordinates.

One vertex (say $\mathbf{Y}_{3}$ ) may be taken at random, and the system of coordinates so chosen that it becomes the point $(1,1,1)$. Thus if cases (i)-(iv) hold, we have for the coordinates of the other vertices

$$
\begin{gathered}
x_{12} x_{23}=x_{13} x_{21}=x_{11} x_{22}, \\
x_{13} x_{22}=x_{12} x_{21},
\end{gathered}
$$

whence

$$
\frac{x_{12}}{x_{13}}=\frac{x_{21}}{x_{23}}=\frac{x_{22}}{x_{21}}=\frac{x_{13}}{x_{11}}=\alpha, \text { say, }
$$

and $Y_{1}, Y_{2}$ are given by $Y_{1}\left(1, \alpha^{2}, \alpha\right), Y_{2}\left(\alpha, \alpha^{2}, 1\right)$, $\alpha$ being arbitrary.

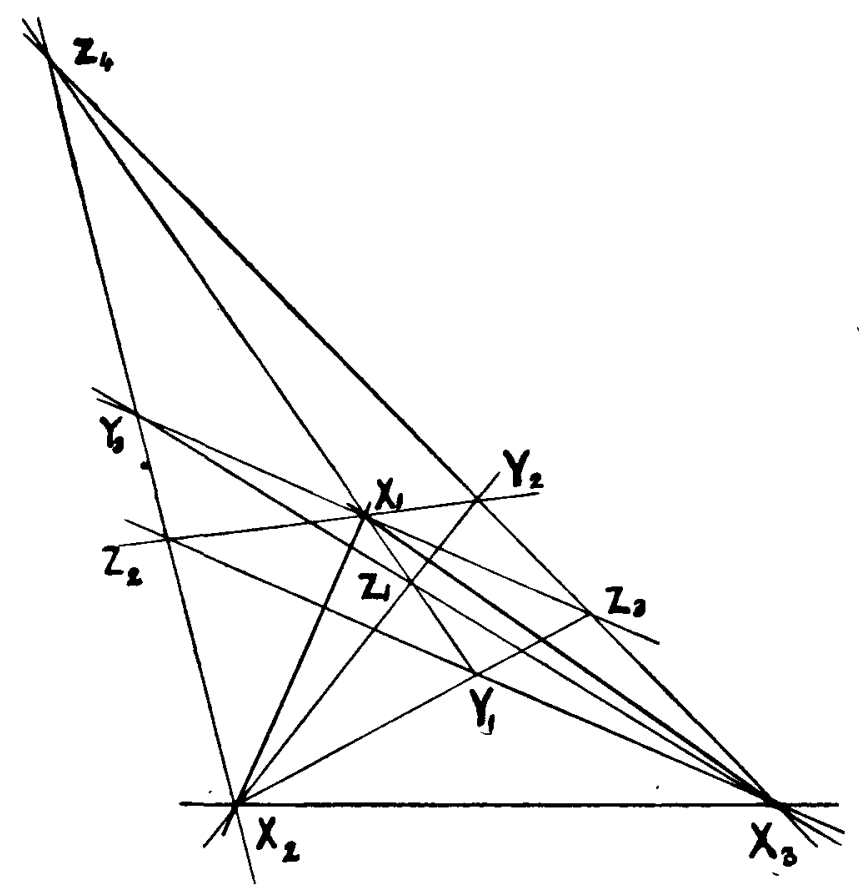

Triangles $\mathrm{X}_{1} \mathrm{X}_{2} \mathrm{X}_{3}, \mathrm{Y}_{1} \mathrm{Y}_{2} \mathrm{Y}_{3}$ in quadruple perspective.

[The figure is given for an arbitrary position of $Y_{3}$, and $\alpha=-2$. The collinearity of $Z_{1} Z_{2} Z_{3}$ is a coincidence, due to the value of $\alpha$ 
chosen. For it is easily found that the coordinates of the poles are

$$
Z_{1}(\alpha, \alpha, 1), Z_{2}\left(1, \alpha^{2}, 1\right), Z_{3}(1, \alpha, \alpha), Z_{4}(1, \alpha, 1) ;
$$

and the determinant of the coordinates of the first three $=\alpha(\alpha-1)^{2}(\alpha+2)$, vanishing for $\alpha=-2$.]

( $\gamma$ ) Sextuple perspective occurs on the fulfilment of all six conditions, but, there being only four independent, one vertex $Y_{3}$ can still be taken at random. The conditions yield

$$
\begin{aligned}
\frac{x_{11}}{x_{13}} & =\frac{x_{12}}{x_{13}}=\frac{x_{13}}{x_{11}}=\frac{x_{22}}{x_{21}}=\frac{x_{23}}{x_{22}}=\frac{x_{21}}{x_{233}} \\
& =\left(\text { clearly) } 1, \omega, \text { or } \omega^{2} .\right.
\end{aligned}
$$

Unity is rejected as reducing $\mathrm{Y}_{1} \mathrm{Y}_{2} \mathrm{Y}_{\text {; }}$ to a point. We take the value $\omega$, and obtain the triangle $\mathrm{Y}_{1}\left(\omega^{2}, \omega, 1\right), \mathrm{Y}_{2}\left(\omega, \omega^{2}, 1\right), \mathrm{Y}_{3}(1,1,1)$; the value $\omega^{2}$ would simply interchange $\mathrm{Y}_{1}, \mathrm{Y}_{2}$. Thus there is only one triangle in sextuple perspective with the triangle of reference, and with a vertex at an arbitrary point. If that point is the real point $(1,1,1)$, the other two vertices are imaginary points on the real line

$$
x_{1}+x_{2}+x_{3}=0 .
$$

\$. Consider the substitution (1) for $n=3$, applied to the system of coordinates here in use. It is evident that $Y_{1} Y_{2} Y_{3}$ is coincident with the invariant triangle of the substitution. We shall therefore revert to our previous notation and call it the triangle $\mathbf{P}_{1} \mathbf{P}_{2} \mathrm{P}_{3}$. The six poles, in the order of $\S \bar{\tau}$, are
(i) $\mathrm{Q}_{3}\left(1,1, \omega^{2}\right)$,
(iv) $\mathbf{R}_{3}(1,1, \omega)$
(ii) $\mathrm{Q}_{2}\left(1, \omega^{2}, 1\right)$,
(v) $R_{2}(1, \omega, 1)$,
(iii) $\mathrm{Q}_{1}\left(\omega^{2}, 1,1\right)$,
(vi) $R_{1}(\omega, 1,1)$.

To pass from one system of homogeneous coordinates to another, with the same triangle of reference, we simply alter the coordinates of each point in definite ratios, so that, e.g. the old coordinates of a point having been $x_{1}, x_{2}, x_{3}$, its new ones are $\left(\alpha x_{1}, \beta x_{2}, \gamma x_{3}\right), \alpha, \beta, \gamma$ being constant for all points. Thus the triangle $\mathrm{T}_{1}\left(\omega^{2} \alpha, \omega \beta, \gamma\right) \mathrm{T}_{2}\left(\omega \alpha, \omega^{2} \beta, \gamma\right) \quad \mathrm{T}_{3}(\alpha, \beta, \gamma)$, whatever the system of coordinates, is in sextuple perspective with the triangle of reference, and can be made to coincide with any triangle in this relation, by giving suitable values to $\alpha: \beta: \gamma$. Now the two sets 
of multipliers $\alpha: \beta: \gamma=\omega^{2}: 1: 1$ and $\omega: 1: 1$ respectively change the points

$\begin{array}{ll} & \mathrm{P}_{1} \mathrm{P}_{2} \mathrm{P}_{3} \mathrm{Q}_{1} \mathrm{Q}_{2} \mathrm{Q}_{3} \mathrm{R}_{1} \mathrm{R}_{2} \mathrm{R}_{3} \\ \text { into } & \mathrm{Q}_{3} \mathrm{Q}_{2} \mathrm{Q}_{1} \mathrm{R}_{1} \mathrm{R}_{3} \mathrm{R}_{2} \mathrm{P}_{3} \mathrm{P}_{1} \mathrm{P}_{2}, \\ \text { and } & \mathrm{R}_{2} \mathrm{R}_{3} \mathrm{R}_{1} \mathrm{P}_{3} \mathrm{P}_{2} \mathrm{P}_{1} \mathrm{Q}_{2} Q_{3} \mathrm{Q}_{2} .\end{array}$

Hence each of the triangles $P_{1} P_{2} P_{3}, Q_{1} Q_{2} Q_{3}, R_{1} R_{2} R_{3}$ is in sextuple perspective with the triangle of reference, and the poles of perspective in each case are the vertices of the remaining two.

\$. The relation of $\mathrm{P}_{1} \mathrm{P}_{2} \mathrm{P}_{3}$ to $\mathrm{X}_{1} \mathrm{X}_{2} \mathrm{X}_{3}$ may be expressed by another geometrical analogy. $A$ point and a line are called harmonic pole and polar with respect to a triangle in their plane when the line, and the joins of the point to the vertices, divide the three sides harmonically. If each vertex and opposite side of one triangle are harmonic pole and polar with respect to another, the former may be called self-polar with respect to the latter; and, as it easily follows from the conditions obtained below that the relation is reciprocal, the two triangles may be called conjugate.

To find the conditions that the three points

$$
\left(x_{a 1}, x_{a 2}, x_{a 3}\right),(\alpha=1,2,3),
$$

form a triangle self-polar with respect to the triangle of reference.

Denoting by $\mathrm{X}_{\alpha \beta}$ the cofactor of $x_{\alpha \beta}$ in the determinant $\Delta$ of the coefficients, and expressing that each point is the harmonic pole of the line joining the other two, we obtain the nine relations

$$
x_{\alpha \beta} \mathrm{X}_{\alpha \beta}=\text { const. }=\frac{1}{3} \Delta \cdot(\mu, \beta=1,2,3) .
$$

The two relations

$$
x_{11} \mathrm{X}_{11}=x_{12} \mathrm{X}_{12}, x_{33} \mathrm{X}_{33}=x_{23} \mathrm{X}_{23}
$$

may be written respectively

$$
\begin{aligned}
& x_{33}\left(x_{12} x_{22}+x_{12} x_{21}\right)=x_{23}\left(x_{12} x_{31}+x_{11} x_{32}\right), \\
& x_{33}\left(x_{11} x_{: 2}-x_{12} x_{21}\right)=x_{23}\left(x_{12} x_{31}-x_{11} x_{32}\right),
\end{aligned}
$$

whence, and by symmetry,

$$
\begin{aligned}
& x_{11} x_{12} x_{33}=x_{23} x_{31} x_{12}=x_{32} x_{13} x_{21}, \\
& x_{11} x_{23} x_{32}=x_{22} x_{31} x_{13}=x_{33} x_{12} x_{21} .
\end{aligned}
$$


These conditions, which satisfy all the nine relations, are exactly those for sextuple perspective (\$7). Thus triangles in sextuple perspective are conjugate, and vice versa.

The triangle $\mathrm{P}_{1} \mathrm{P}_{2} \mathrm{P}_{3}$, being uniquely determined as soon as the system of coordinates is specified, might be fitly called the principal related triangle for the system.

$\S 10$. The substitution (1) becomes for $n=3$,

$$
\left.\begin{array}{l}
x_{1}^{\prime}=a_{1} x_{1}+a_{2} x_{2}+a_{3} x_{3} \\
x_{2}^{\prime}=a_{3} x_{1}+a_{1} x_{2}+a_{2} x_{3} \\
x_{3}^{\prime}=a_{2} x_{1}+a_{3} x_{2}+a_{1} x_{3}
\end{array}\right\}
$$

Taking $x_{1}, x_{2}, x_{3}$ as homogeneous point-coordinates, we must distinguish two possible interpretations. First, it might mean that the point whose coordinates are $x_{1}, x_{2}, x_{3}$ has to be shifted to $\left(x_{1}^{\prime}, x_{2}^{\prime}, x_{3}^{\prime}\right)$; thus $(1,0,0)$ would be shifted to $\left(a_{1}, a_{3}, a_{2}\right)$, etc. Secondly, it might mean that the line whose equation is

$$
l_{1} x_{1}+l_{2} x_{2}+l_{3} x_{3}=0
$$

is to be shifted into the position of the line

$$
\begin{gathered}
l_{1} x_{1}^{\prime}+l_{2} x_{2}{ }^{\prime}+l_{3} x_{3}^{\prime} \\
\equiv\left(a_{1} l_{1}+a_{3} l_{2}+a_{2} l_{3}\right) x_{1}+\left(a_{2} l_{1}+a_{1} l_{2}+a_{3} l_{3}\right) x_{2}+\left(a_{3} l_{1}+a_{2} l_{2}+a_{1} l_{3}\right) x_{3}=0 ;
\end{gathered}
$$

so that $x_{1}=0$ would become

and so on.

$$
a_{1} x_{1}+a_{2} x_{2}+a_{3} x_{3}=0
$$

If iǹ (13) we replace $a_{1}, a_{2}, a_{3}$ respectively by $A_{1}, A_{3}, A_{2}$ the cofactors of $a_{1}, a_{3}, a_{2}$ in the determinant of the coefficients, the first interpretation of the new substitution is geometrically equivalent to the second interpretation of (13). We can therefore afford to ignore the second interpretation as virtually involved in the first. It is also easy to see that the geometrical effects of (13) in its two interpretations are mutualiy inverse.

$\$ 11$. With the first interpretation, then, (13) shifts $\mathrm{X}_{1} \mathrm{X}_{2} \mathrm{X}_{3}$ into $\mathbf{Y}_{1}\left(a_{1}, a_{3}, a_{2}\right), \mathbf{Y}_{3}\left(a_{2}, a_{1}, a_{3}\right), \mathbf{Y}_{2}\left(a_{3}, a_{3}, a_{1}\right)$. These coordinates satisfy equations (A) of $\$ 7$; hence every triangle into which $\mathrm{X}_{1} \mathrm{X}_{2} \mathrm{X}_{3}$ is changed by (13) is in triple perspective with $\mathrm{X}_{1} \mathrm{X}_{2} \mathrm{X}_{3}$ itself. 
The poles are found to be

$$
Z_{1}\left(a_{1}^{-1}, a_{2}^{-1}, a_{3}^{-1}\right), Z_{2}\left(a_{3}^{-1}, a_{1}^{-1}, a_{2}^{-1}\right), Z_{3}\left(a_{2}^{-1}, a_{3}^{-1}, a_{1}^{-1}\right),
$$

satisfying equations $(B)$ of $\$ 7$, and therefore forming a triangle in triple perspective with $X_{1} X_{2} X_{3}$, but in reversed cyclic order.

The condition that $Y_{1}, Y_{2}, Y_{3}$ should satisfy (iv) in addition to (i)-(iii) is

$$
a_{2}{ }^{3}=a_{3}{ }^{3} ;
$$

thus the equality of two coefficients in the substitution involves quadruple perspective between $\mathrm{X}_{1} \mathrm{X}_{2} \mathrm{X}_{3}$ and every triangle obtained from it by the substitution; and the same holds if two coefficients have the ratio $\omega$ or $\omega^{2}$, though the triangle is then imaginary. Whatever the system of point-coordinates and the value of $\alpha$, the triangle $(\alpha, 1,1),(1,1, \alpha),(1, \alpha, 1)$ is in quadruple perspective in ways (i) to (iv) with $X_{1} X_{2} X_{3}$.

$\$ 12$. The invariant points and lines of (13), forming the invariant triangle, are

$$
\left.\begin{array}{ll}
\mathbf{P}_{1}\left(\omega^{2}, \omega, 1\right), & \sqrt{ } 3 \cdot \xi_{1}=\omega x_{1}+\omega^{2} x_{2}+x_{3} \\
\mathbf{P}_{2}\left(\omega, \omega^{2}, 1\right), & \sqrt{ } 3 \cdot \xi_{2}=\omega^{2} x_{1}+\omega x_{2}+x_{3} \\
\mathbf{P}_{3}(1,1,1), & \sqrt{ } 3 \cdot \xi_{3}=x_{1}+x_{2}+x_{3}
\end{array}\right\}
$$

This triangle is what we have called $(\S 9)$ the principal related triangle for the particular system of point-coordinates in use. It is in sextuple perspective with, and conjugate to, the triangle of reference $(\$ \$ 8,9)$; and not only so, but, as perspective and harmonic properties are unaltered by a linear transformation, it stands in the same relations to every triangle obtainable by (13) from the triangle of reference. When we say "by (13)," we mean by every substitution of the form (13), the result being independent of the coefficients of (13).

\$13. The geometrical interpretation of $S(\rho)$ for $n=3$, or of $\mathrm{S}(\omega)$, is plain : it changes the triangle of reference $\mathrm{X}_{1} \mathrm{X}_{2} \mathrm{X}_{3}$ into the principal reluted triangle $\mathrm{P}_{1} \mathrm{P}_{2} \mathrm{P}_{3}$ for the coordinates in use, the order 'of the vertices being significant. $S\left(\omega^{2}\right)$ will change $X_{1} X_{2} X_{3}$ into $P_{2} P_{1} P_{3}$, and substitutions of like form will change $X_{1} X_{2} X_{3}$ into each of the other four ways in which $\mathrm{P}_{1} \mathrm{P}_{2} \mathrm{P}_{3}$ may be named. Each pair of substitutions, such as $S(\omega)$ and $S\left(\omega^{2}\right)$, gives one invariant triangle, and the nine vertices are as follows, the three 
last deduced from the table of $\$ 5$, the others by cyclic interchange :-

$$
\begin{array}{lll}
\mathbf{S}_{11}(0,1,-1), & \mathbf{S}_{12}(\sqrt{ } 3+1,1,1), & \mathbf{S}_{13}(-\sqrt{ } 3+1,1,1) ; \\
\mathbf{S}_{21}(1,-\sqrt{ } 3+1,1), & \mathbf{S}_{22}(-1,0,1), & \mathbf{S}_{33}(1, \sqrt{ } 3+1,1) ; \\
\mathbf{S}_{31}(1,1, \sqrt{ } 3+1), & \mathbf{S}_{32}(1,1,-\sqrt{ } 3+1), & \mathbf{S}_{33}((1,-1,0) .
\end{array}
$$

They are all real, the three diagonal ones lying in a straight line, and the others, in two sets of three, forming triangles in triple perspective with $\mathrm{X}_{1} \mathrm{X}_{2} \mathrm{X}_{3}$.

Under repetitions of $S(\omega), X_{1} X_{2} X_{3}$ passes through the successive stages $\mathrm{P}_{1} \mathrm{P}_{2} \mathrm{P}_{3}, \mathrm{X}_{2} \mathrm{X}_{1} \mathrm{X}_{3}, \mathrm{P}_{2} \mathrm{P}_{1} \mathrm{P}_{3}$, and so back to $\mathrm{X}_{1} \mathrm{X}_{2} \mathrm{X}_{3}$, the points $S_{31}, S_{32}, S_{33}$ remaining fixed ; and similarly in the other cases.*

* Since writing the above, I have found the following references to triangles in multiple perspective :-

Homologous Triangles, by Thomas Muir, M.A. [Mess. Math. 2 (1873)]. "Triply homologous" triangles are discussed, the vertices being taken in the same cyclic order throughout.

Perspective Dreiecke und Tetraeder, by Edmund Hess [Math. Annalen 28 (1887)]. Here sextuply perspective triangles are introduced, the standpoint being grometrical, and the application different from that of the present paper. 